\title{
THE JUDGE AND THE RIGHT OF PREEMPTION LAND IN CAMEROON
}

\section{(Le Juge Et Le Droit De Préemption Foncière Au Cameroun¹)}

\author{
Désiré Aurèle Mbang Essono
}

Doctorant en droit privé à l’Université de Dschang (Cameroun)

Email : mbangdesire@yahoo.com

Cite this article:

Désiré Aurèle Mbang Essono (2021), The Judge and The Right of Preemption Land in Cameroon. African Journal of Law, Political Research and Administration 4(1), 52-69. DOI: $10.52589 / A J L P R A-$ I8WUOUR1.

\section{Manuscript History}

Received: 10 March 2021

Accepted: 10 April 2021

Published: 14 May 2021

Copyright $($ C) 2020 The Author(s). This is an Open Access article distributed under the terms of Creative Commons AttributionNonCommercial-NoDerivatives 4.0 International (CC BY-NC-ND 4.0), which permits anyone to share, use, reproduce and redistribute in any medium, provided the original author and source are credited.
RESUME : L'action du juge dans le cadre de la préservation de la propriété foncière confrontée au droit de préemption demeure très appréciable. En effet, grâce à l'accroissement de son domaine de compétence, le juge administratif a été érigé en protecteur à part entière des biens des personnes privées soumis à la préemption. L'ordre juridique camerounais réserve une place traditionnelle au juge judiciaire au sujet de la protection de la propriété foncière préemptée, car il contribue par son office à contrer toute atteinte dont la propriété foncière pourrait être victime. Le juge judiciaire aurait pu voir son champ de compétence s'élargir avec la possibilité qui lui a été reconnue d'effectuer le contrôle de légalité des opérations de préemption foncière, mais cette attribution de compétence demeure très clairement encadrée.

Mots clés: Juge, droit de préemption, propriété foncière, détermination, compétence.

ABSTRACT: The action of the Cameroonian judge in the context of the preservation of land ownership faced with the right of pre-emption remains very marginal. On the other hand, thanks to the increase in its field of competence, the administrative judge has been erected as a full-fledged protector of the property of private persons subject to preemption. Through his action, the administrative judge should ensure that land pre-emption operations comply with the law. In the event of non-compliance with legality, the respondent could be held liable. Moreover, the Cameroonian legal system reserves a traditional place for the judicial judge in the protection of pre-empted land ownership, as he contributes through his office to counter any infringement of land ownership. The judicial judge's field of competence could have been broadened with the possibility of carrying out legality control of land pre-emption operations, but this attribution of competence remains very clearly defined.

KEYWORDS: Judge, Right of Pre-Emption, Land Ownership, Determination, Competence.

\footnotetext{
${ }^{1}$ Nous tenons à remercier le Professeur I-L MIENDJIEM pour la lecture, et ses remarques émises en vue d'améliorer la qualité de cet article de doctrine.
} 


\section{INTRODUCTION}

L'attention accordée à la terre est la preuve s'il en était encore besoin de la place de choix qu'elle occupe dans tout processus de planification du développement. Aujourd'hui comme hier, il apparait que la terre est au cœur de toutes les convoitises avec des enjeux socioéconomiques $^{2}$ et culturels ${ }^{3}$. Cet apport stratégique de la terre à l'essor économique du Cameroun a amené les pouvoirs publics à se doter des moyens juridiques facilitant l'accès à des espaces fonciers ${ }^{4}$. C'est ainsi que le législateur a reconnu à l'Etat, mais aussi aux collectivités territoriales décentralisées, la possibilité de recourir à une technique particulière d'acquisition des espaces fonciers, à savoir, le droit de préemption. Cet instrument d'appropriation foncière a depuis longtemps fait son chemin dans le système juridique camerounais. Cette consécration est réalisée dans de nombreux textes pour le moins éparses, anciens ou contemporains.

De façon chronologique ${ }^{5}$, on notera que par le décret du 21 juillet 1932 , le législateur institue au Cameroun le régime foncier de l'immatriculation ${ }^{6}$, à travers ce texte, le droit de préemption est consacré relativement aux immeubles immatriculés en son article 23, puisqu'il est reconnu à tout copropriétaire indivis d'un même immeuble, à tout cohéritier sur les immeubles de la succession, à tout copropriétaire divis d'une maison d'habitation, au superficiaire pour l'acquisition du sol et au propriétaire du sol pour l'acquisition de la superficie, d'acquérir la portion vendue à un tiers en se substituant à cet acquéreur, moyennant le remboursement du prix de vente, y compris les loyaux coûts du contrat, et s'il y’a lieu des dépenses engagées pour amélioration. Plus tard, un décret n ${ }^{\circ} 68-61-C O R$ du 30 avril 1968 relatif aux zones à urbaniser par priorité ${ }^{7}$ va prévoir en son article 3 un droit de préemption à l'endroit de la commune ou d'un organisme concessionnaire en cas de vente d'un terrain situé dans la zone à urbaniser par priorité. L'ordonnance $n^{\circ} 74-1 \mathrm{du} 06$ juillet

\footnotetext{
${ }^{2}$ La possession du terrain en ville représente pour les citadins une preuve de réussite sociale. En effet, avoir un terrain est considéré comme un signe extérieur de richesse. Avoir accès à une propriété foncière en ville revient à consolider son assise économique, à renforcer son train de vie, à assurer une certaine sécurité à sa descendance. Cette considération sociale est d'autant plus forte que posséder un espace (même non bâti) en ville devient une obsession pour de nombreuses personnes. La terre est, en ce sens, un bien marchand plus que prisé. Voir. R-J. ASSAKO ASSAKO et H-G. NJOUONANG DJOMO, "Curée foncière et stratégies d'accès à la terre dans la périphérie sud-ouest de Yaoundé », Syllabus Review Human \& Social Science Séries 6 (1), 2015, p. 16.

${ }^{3}$ Dans presque tous les quartiers populaires d'habitat précaire de Yaoundé en général et de Yaoundé VI en particulier, la raison primordiale d'occupation de l'espace repose d'abord sur des considérations traditionnelles selon lesquelles la terre est un héritage ancestral qu'on peut occuper librement ou coutumièrement, sans devoir obtenir l'autorisation de l'État. C'est ce qui explique que plus de $53 \%$ des « propriétaires » enquêtés ne possèdent pas de titre foncier, avec plus de la moitié qui estiment n'en avoir pas besoin, du fait de leur droit coutumier sur ladite parcelle. R-J. ASSAKO ASSAKO et H-G. NJOUONANG DJOMO, «Curée foncière et stratégies d'accès à la terre dans la périphérie sud-ouest de Yaoundé », op cit, p. 17.

${ }^{4}$ Voir. Désiré-Aurèle. MBANG ESSONO, Le droit de préemption en matière foncière : Réflexions à partir des droits camerounais et français, Thèse de Doctorat droit privé en préparation, Université de Dschang, 2020, p. 2.

${ }^{5}$ Ils sont nombreux les textes juridiques qui évoquent d'une manière ou d'une autre le concept de droit de préemption, c'est le cas notamment de l'article 21 alinéa (2) de la loi n 2000 / 010 du 19 décembre 2000 régissant les archives qui reconnait au profit de l'Etat et aux collectivités territoriales décentralisées le droit de disposer d'un droit de préemption sur tout document d'archives privées mis en vente publique. Consacré en faveur des communautés villageoises riveraines des zones forestières du Cameroun par la loi n 94/01 du 20 janvier 1994 portant régime des forêts, le droit de préemption vise à créer des forêts communautaires que vont gérer des populations rurales. Ce droit s'appuie sur des bases juridiques qui relèvent aussi bien de la Constitution du Cameroun que d'un arsenal législatif et réglementaire.

${ }^{6} \mathrm{Ce}$ texte demeure toujours en vigueur, cela est perceptible à partir de la lecture de l'arrêt de la cour suprême ${ }^{\circ} 5 / \mathrm{L}$ du 26 octobre, inédit. Cité in G.B. DZEUKOU, observations sur Cour Suprême, Arrêt n²49/Com du 06 novembre 2008, affaire Société ARNO Sarl C/Société Industrielle de produits chimiques et aromatiques (SIPCA) et la Société SAPI SCI., Juridis Périodique n86, Avril-Mai-Juin 2011, p. 50.

${ }^{7}$ J.O.C.O.R., 1968, p. 353 ; RCDC., T. VIII, pp. 103-104.
} 
1974 fixant le régime foncier ${ }^{8}$ accorde pour sa part un «droit de préférence » aux occupants de bonne foi en cas de revente des immeubles habités, urbains ou ruraux, tombés dans le domaine national en raison de la déchéance qui a frappé les titulaires des droits réels qui ont négligé de transformer ceux-ci en titres fonciers ${ }^{9}$; et, un droit de préemption à l'Etat en cas de revente d'un immeuble acquis par des personnes physiques ou morales de nationalité étrangère ${ }^{10}$. Le décret $\mathrm{n}^{\circ} 76-167$ du 27 avril 1976 fixant les modalités de gestion du domaine privé de $1^{\prime}$ Etat ${ }^{11}$, confère également un droit de préemption à l'Etat à l'expiration du bail ordinaire ou emphytéotique «sur tous les aménagements, construction et installations» réalisés sur l'immeuble. Plus récemment, la loi de $2004^{12}$ reconnaitra à l'Etat mais aux collectivités territoriales décentralisées un droit de préemption pouvant leur permettre d'acquérir des espaces fonciers.

Le droit de préemption est défini par l'article 86 du code camerounais de l'urbanisme comme « un droit qui permet à la puissance publique de se porter acquéreur prioritaire d'un bien immobilier qu'un propriétaire désire vendre, s'applique dans les zones où la puissance publique souhaite s'assurer de la maîtrise du sol, veut contrôler l'évolution des prix fonciers ou acquérir certains immeubles bâtis ou non bâtis, sans toutefois avoir recours à la procédure d'expropriation.». Cette prérogative de puissance publique peut être aussi considérée comme "la faculté conférée par la loi ou par la convention à une personne d'acquérir, de préférence à toute autre, un bien que son propriétaire se propose de céder, en se portant acquéreur aux prix et conditions de la cession projetée ${ }^{13}$.

Les facilités qu'offre l'usage du droit de préemption foncière, ne sauraient faire oublier les abus dont peuvent être victimes les propriétaires des espaces fonciers soumis à la préemption. En effet, l'histoire jurisprudentielle du droit de préemption foncière nous révèle que cet instrument a été, et, est à l'origine des atteintes multiples au droit de propriété mais aussi à la liberté contractuelle. En vue de préserver le caractère quasi absolu du droit de propriété ${ }^{14}$ face à la toute-puissance du droit de préemption foncière, le législateur a mis en place un certain nombre de mécanismes juridiques pouvant amenuiser les multiples abus. L'atténuation des atteintes portées au droit de propriété par la préemption foncière a consisté à mettre en place un contrôle juridictionnel diversifié dont l'objectif est de veiller au respect des règles qui encadrent la préemption foncière.

\footnotetext{
${ }^{8}$ J.O.R.U.C., 05 aout 1974, ${ }^{\circ}$ 1, supplémentaire, pp. $2-5$; R.C.D.C. ; 1974 ; T.XIV (B), pp. 582-585 ; R.C.D/C.L.R., 1975, $\mathrm{n}^{\circ} 7$, pp. 82-85.

${ }^{9} \mathrm{Cf}$. Article 5.

${ }^{10}$ Voir article 10 de l'ordonnance $n^{\circ} 74-1$ du 06 juillet 1974 fixant le régime foncier au Cameroun.

${ }^{11}$ J.O.R.U.C., $1^{\text {er }}$ mai $1976, \mathrm{n}^{\circ} 1$, supplémentaire, pp. 13-19.

${ }^{12}$ Loi n²004/003 du 21 avril 2004 portant Code d'urbanisme en République du Cameroun.

${ }^{13}$ Gérard. CORNU, Association Henri Capitant - Vocabulaire juridique, 9ème éd, 2011, p. 235.

${ }^{14}$ Consacré par la Déclaration des Droits de l'Homme et du Citoyen de 1789 aux cotés de la liberté et de la sûreté (Article 2 de la DDHC : « le but de toute association politique est la conservation des droits naturels et imprescriptibles de l'homme. Ces droits sont la liberté, la propriété, la sûreté et la résistance à l'oppression »), le droit de propriété est conçu comme l'un des socles de toute société. Concept fondamental, il fait aujourd'hui l'objet d'une protection constitutionnelle qui se superpose à celles traditionnellement mises en œuvre par le juge judiciaire, gardien de la propriété privée, et par le juge administratif dont le rôle a pu à cet égard, paraitre subsidiaire. M. VERPEAUX note à cet égard que si « le juge judicaire est souvent présenté comme le juge de la propriété, cette certitude mérite d'être revisitée ». M. VERPEAUX, «le juge administratif gardien du droit de propriété », RFD adm. 2003, p. 1096.), mais au combien important. L'apport des juges administratifs et judiciaires à la protection de certains droits fondamentaux du propriétaire de l'espace foncier préempté n'est pas à négliger car, à travers le contrôle des procédures de mise en œuvre du droit de préemption mais aussi grâce aux sanctions que peuvent prononcer ses juges à l'égard de l'Etat ou collectivités territoriales décentralisées, les dits droits pourront s'en trouver davantage garantis. C'est donc dire, qu'au moyen de la facilité reconnue au propriétaire du bien préempté de recourir au juge, il est clair que ces droits seront mieux protégés. Ce recours au juge participe de la garantie effective des droits en général, et du droit de propriété en particulier
} 
La consécration d'un contrôle juridictionnel n'évacue pas toutes les difficultés. L'une des principales difficultés est la détermination du juge compétent en matière de protection de la propriété foncière confrontée au droit de préemption. En droit, la question se pose de savoir quel est le juge compétent en matière de préemption foncière au Cameroun. La détermination du domaine de compétence du juge administratif demeure sujette à controverse puisqu'en droit comparé, le Conseil d'Etat français suggérait dans un rapport sur le droit de préemption que le juge administratif devrait être le seul compètent à statuer tant sur la décision de préemption que sur les contrats y découlant. Appréciant cette idée, la doctrine soutient que, si cette proposition est formellement consacrée, elle serait un facteur certain de simplification du contentieux de la préemption foncière. La compétence du juge administratif ou judiciaire devrait se déterminer en fonction de la nature de l'acte visé par le recours, or la qualification des actes nécessaires à la procédure de préemption n'est pas chose aisée en raison de l'enchevêtrement de règles tantôt privatistes tantôt administrativistes. ${ }^{15}$ Pour la doctrine, le juge administratif devrait être compétent pour trancher les questions de la régularité administrative de la décision de préemption elle-même, le juge judiciaire étant appelé à connaitre de toutes les questions relatives notamment aux obligations de paiement et de consignation et aux conséquences de l'annulation d'une décision de préemption ${ }^{16}$. Malgré cette répartition doctrinale des règles de compétence, le flou persiste et révèle la nécessité d'une délimitation nette entre le domaine de compétence du juge administratif et celui du juge judiciaire en matière de préemption foncière en droit camerounais. À défaut, cette concurrence serait préjudiciable pour les propriétaires qui ne sauraient pas vers qui se tourner, et pour les juges qui se demanderaient s'ils sont ou non compétents ${ }^{17}$. Si le juge judiciaire est considéré comme le gardien traditionnel du droit de propriété, le juge administratif doit l'être comme son gardien contemporain ${ }^{18}$. En observant de plus près la jurisprudence, il est loisible de se rendre compte que la tendance est à l'accroissement du champ de compétence du juge administratif (I). En revanche, l'on constate un cantonnement du domaine de compétence du juge judiciaire dans le cadre du contrôle du droit de préemption foncière (II).

\section{I) L'accroissement du champ de compétence du juge administratif dans le cadre du contrôle du droit de préemption foncière}

La décision de préemption illégale peut être contestée devant le juge administratif par un requérant ayant intérêt pour agir, par exemple une personne détenant un droit de préférence pour l'acquisition de l'immeuble préempté ${ }^{19}$, ou un fermier exploitant le terrain préempté20. Cette admission du juge administratif comme acteur majeur du contrôle des opérations de préemption n'a pas toujours été un long fleuve tranquille, puisque c'est à la suite d'âpres batailles que le juge administratif finira par être reconnu à l'instar de son homologue le juge judiciaire comme un gardien de la propriété foncière (A). Passé l'étape de l'adoubement du

\footnotetext{
15 Jessica. GRISSIER, L'élargissement du droit de préemption urbain aux biens commerciaux et artisanaux : L'étude d'un instrument d'intervention public dans la sphère commerciale, Thèse de doctorat de droit privé, Université de Toulouse 1 Capitole, 2015, p. 280.

${ }^{16}$ Pour avoir une illustration récente de la compétence judiciaire en matière de rétrocession, CE 7 janvier 2013, 80 ; RDI 20113. Obs P. Soler-couteaux, AJCT 2013 ; S. DEVIDAS, « Le droit de préemption sur les espaces naturels sensibles, outil privilégié de la protection de l'environnement », Actualité Juridique Collectivités Territoriales, Mai 2013, p. 231.

${ }^{17}$ Michel. ROLAIN, Les limitations au droit de propriété en matière immobilière, Thèse de Doctorat en droit, Université de Nice Sophia Antipolis, 30 novembre 2015, p. 15

${ }^{18}$ B. MALLET-BRICOUT et N. REBOUL-MAUPIN, Droit des biens, juin 2013-juin 2014, p. 1844 : « Gardiens naturels de la propriété privée, les tribunaux judiciaires avaient jusqu'alors reçu la fonction traditionnelle d'assurer la protection légale du droit de propriété ».

${ }^{19}$ Conseil d'État, 20 mars 1991, Roucaute, Rec. T. p. 1109.

${ }^{20}$ Cour adm appel Lyon, 7 juillet 1995, M. et Mme Mondolini, Rec. p. 539.
} 
juge administratif, il pourrait tout aussi effectuer le contrôle de la légalité des décisions de préemption foncière $(\mathrm{B})$.

\section{Le juge administratif, juge protecteur de la propriété foncière confrontée au droit de préemption}

La consécration du juge administratif comme gardien de la propriété foncière a été une longue marche (1), en dépit de ce chemin aussi long, le juge administratif est dorénavant armé pour assurer la protection de la propriété privée (2).

\section{1) La longue marche vers la consécration du juge administratif comme gardien de la propriété foncière}

Pendant longtemps, le travail de protection juridictionnelle de la propriété privée avait toujours été considéré comme étant la chasse gardée du juge judiciaire, car celui-ci était accrédité d'un minimum d'indépendance pouvant garantir sa mission de juger les autorités publiques accusées de porter des atteintes sur des propriétés foncières appartenant à des particuliers. Dans l'optique de participer à la protection de la propriété privée, le juge administratif va s'ériger en protecteur à part entière des biens des personnes privées. Il faut dire que cette avancée a été un travail de longue a laine car, il fallait déjà faire table rase des idées reçues sur la probable proximité qu'il pourrait y avoir entre le juge administratif et l'administration dont il est appelé à juger pour l'admettre comme un juge pouvant contribuer à la préservation de la propriété privée. L'indépendance et l'impartialité du juge administratif avaient souvent été questionnées, et même interrogées. En effet, comment ce juge, émanation de l'administration aux ordres de l'exécutif serait-il à même de protéger la propriété privée face aux assauts de cette administration? En taché de ce «péché originel $\gg^{21}$, le juge administratif va cependant faire preuve d'impartialité et conquérir, année après année, une indépendance que le Conseil constitutionnel viendra consacrer en $1980^{22}$. C'est ainsi que, le juge administratif, grâce à «la montée en puissance des aspirations libérales et individuelles et les mouvements d'européanisation et de constitutionnalisation des droits fondamentaux vont l'amener à développer une jurisprudence protectrice de ces droits et notamment le droit de propriété. Le contrôle de conventionalité des actes administratifs, et par la suite, mais de façon plus indirecte, la question prioritaire de constitutionnalité ont ainsi conduit le juge administratif à veiller à la protection de ce droit fondamental qu'est le droit de propriété ${ }^{23}$. Le Conseil d'État considérant par exemple que la compétence judiciaire en matière d'atteinte au droit de propriété était un principe général du droit ${ }^{24}$, le Conseil constitutionnel a refusé de consacrer constitutionnellement une conception aussi générale. Par ce raisonnement juridictionnel, le juge administratif bénéficiera désormais d'une compétence pouvant lui permettre de veiller à la protection du droit de propriété en général et dans la cadre de l'exercice et de la mise en œuvre du droit de

\footnotetext{
${ }^{21}$ J. RIVERO, « Dualité de juridictions et protection des libertés », Revue Française de Droit Administratif, 1990, La dualité de juridictions en France et à l'étranger, Bicentenaire de la loi des 16-24 aout 1790, p. 735 : «Création napoléonienne, la juridiction administrative a longtemps été entaché aux yeux des libéraux de ce péché originel : œuvre d'un pouvoir autoritaire, elle ne pouvait, pensaient-ils, que le servir, et il eut été illusoire d'elle qu'elle puisse assurer contre lui la protection des libertés ». Cité in S. PAVAGEAU, «La propriété privée à l'épreuve du dualisme juridictionnel », in Entre les ordres juridiques, Mélanges en l'honneur du Doyen Francois HervouËt, LGDJ, pp. 665-680, nov 2015, p. 675.

${ }^{22}$ Cons. Const., n॰80-119, DC, 22 juillet 1980, Loi portant validation d'actes administratifs. Rec. p. 46, Grandes décisions, $\mathrm{n}^{\circ} 29$, Validation d'actes administratifs ; Carcassonne, AJDA. 1980, p. 602.

${ }^{23}$ S. PAVAGEAU, « La propriété privée à l'épreuve du dualisme juridictionnel », article précité, pp. 665-680.

${ }^{24}$ CE, 19 févr. 1960 Féd. Algérienne des syndicats de défense des irrigants. - V. également T. confl, 18 déc. 1947, Sieur Morin c/ Cortesi : Lebon, p. 515.
} 
préemption foncière en particulier. En droit camerounais, la loi n ${ }^{\circ}$ 2006/022 du 29 décembre 2006 fixant l'organisation et le fonctionnement des tribunaux administratifs a clairement disposé à l'article 2 alinéa 2 que, "les tribunaux administratifs connaissent en premier ressort, du contentieux administratif concernant l'Etat, les collectivités territoriales décentralisées et les établissements publics administratifs, sous réserve des dispositions de l'article 14 (2), de la présente loì. Etant entendu que l'accomplissement des opérations de préemption foncière au Cameroun est de la compétence de l'Etat et des collectivités territoriales décentralisées, il est donc claire que tout différend qui pourrait naître de cette activité devrait être réglé par le juge administratif.

En droit comparé du contentieux de la préemption foncière, le problème a trouvé une esquisse de solution consistant pour le législateur français a clairement reconnaitre la compétence du juge administratif pour régler des litiges nés de l'exercice ou de la mise en œuvre du droit de préemption foncière. En effet, le contentieux administratif en général comprend les recours en annulation pour excès de pouvoir et, en matière répressive, les recours incidents en appréciation de la légalité notamment les actions en indemnisation du préjudice causé par un acte administratif, les litiges concernant les contrats (à l'exception de ceux conclus même implicitement sous l'empire du droit privé) ou les concessions de services publics notamment. En matière de contentieux de la préemption, deux types de recours peuvent être formés devant le juge administratif, un recours pour excès de pouvoir tendant à l'annulation des actes administratifs ou un recours de plein contentieux. Tout d'abord, s'agissant du recours pour excès de pouvoir, il pourra être exercé soit à l'encontre de la décision instaurant le droit de préemption urbain sur le territoire de la commune soit à l'encontre de la délibération autorisant la préemption. Dans ce cas, le requérant devra répondre aux conditions de droit commun posées en matière de procédure administrative. Il devra donc former son recours dans le délai de droit commun de deux mois ${ }^{25}$ à compter de la publication de la délibération litigieuse ainsi que justifier d'un intérêt lui donnant qualité à agir. Ont ainsi pu être considérés comme ayant un intérêt le vendeur de l'immeuble soumis à la préemption ${ }^{26}$.

Dans le prolongement du renforcement de l'idée d'une compétence du juge administratif en matière de garantie du droit de propriété face à la préemption foncière. L'on affirmera sans ambages que les décisions par lesquelles le titulaire du droit de préemption exerce son droit ou renonce à le mettre en œuvre sont des décisions qui sont prises par l'administration dans l'exercice de ses prérogatives de puissance publique. Et, en vertu du principe de séparation des autorités administratives et judiciaires consacré par la loi des 16-24 août 1790 et le décret du 16 fructidor an III, la juridiction administrative est par conséquent seule compétente pour connaître des recours tendant à l'annulation ou à la réformation de ces actes, mais aussi pour statuer, le cas échéant par voie de question préjudicielle, sur toute contestation de leur légalité, soulevée à l'occasion d'un litige relevant à titre principal de l'autorité judiciaire ${ }^{27}$. À première vue, il semblerait qu'il s'agisse d'un acte de droit privé faisant partie des documents internes de la phase contractuelle entre le titulaire du droit de préemption et le vendeur. En effet, il est possible que l'intervention des juridictions administratives soit perçue comme une sorte d'intrusion dans un domaine relevant traditionnellement des tribunaux judiciaires, gardiens de la propriété privée et juges naturels

\footnotetext{
${ }^{25}$ Article R. 421-1 du Code de justice administrative.

${ }^{26} \mathrm{CE}, 21$ mai 2008, Commune de Houilles, req. $\mathrm{n}^{\circ} 296156$.

${ }^{27} \mathrm{CE}, 30$ déc. $2013, \mathrm{n}^{\circ} 365610$.
} 
de l'institution classique du droit civil que représente la préemption. Mais en réalité, à l'heure actuelle, il est clairement établi que l'acte par lequel le titulaire ou le délégataire du droit de préemption décide d'acquérir un bien présente le caractère d'un acte administratif comportant l'utilisation d'une prérogative de puissance publique dans un intérêt général. Tous ses arguments juridiques concourent à la consécration du juge administratif comme gardien à part entière de certains droits du propriétaire de l'espace foncier préempté à l'instar du droit de propriété.

\section{2) Le juge administratif dorénavant armé pour assurer la protection de la propriété foncière}

Après la période de grande incertitude au sujet de la compétence du juge administratif à jouer le rôle majeur de gardien de la propriété foncière face au droit de préemption, le législateur a opté dorénavant pour une reconnaissance du rôle de ce juge en la matière. Cette œuvre d'adoubement s'est faite par la fourniture des outils devant permettre à ce juge d'assumer la protection effective de la propriété privée contre les éventuelles atteintes dont elle pourrait subir du fait de l'action de l'administration préemptrice. Pour illustrer l'action du juge administratif dans le cadre de la protection de la propriété foncière confrontée au droit de préemption, l'on est conduit à prendre quelques exemples de cas de jurisprudence où le juge $\mathrm{y}$ a joué sa partition. En effet, il s'était longtemps posé le problème de la détermination des personnes pouvant saisir la justice pour constater la nullité d'une procédure de préemption foncière illégale. Pour apporter des éléments de réponse à cette difficulté, le juge administratif affirme par exemple que « seuls en principe justifient un intérêt suffisant pour contester une décision de préemption ceux dont le projet est directement contrarié par cette décision, à savoir le vendeur du bien préempté ainsi que l'acquéreur évincé, sous réserve que ce dernier prouve qu'il avait bien cette qualité au moment où la décision de préemption est intervenue $»^{28}$. Bien plus, l'acquéreur évincé, qui bénéficiait à la date à laquelle le droit de préemption a été exercé, d'une promesse de vente sur l'immeuble, justifie-t-il d'un intérêt lui donnant qualité pour agir contre la décision de préemption, et ceci quand bien même la promesse de vente serait devenue caduque postérieurement à la décision litigieuse ${ }^{29}$, faute pour l'acquéreur de s'être acquitté de toutes ses obligations envers le propriétaire ${ }^{30}$. Cette caducité n'exclut pas en effet qu'en cas d'annulation de la décision de préemption, le propriétaire et l'acquéreur évincé conviennent de poursuivre la vente, ce qui explique que l'intérêt à agir de l'acquéreur ait ici été reconnu. La jurisprudence reconnait aussi d'une manière générale qu'ont un intérêt direct à agir le requérant dont l'intention d'acquérir la parcelle ressort manifestement d'une lettre adressée au maire ainsi que des mentions portées sur la déclaration d'intention d'aliéner établie par le notaire chargé de la vente, ou le requérant qui, sans être titulaire d'une promesse de vente, était seulement pressenti pour acquérir le bien, ou encore les acquéreurs initiaux du bien, alors même qu'ils auraient, par un nouvel acte passé avec l'accord du vendeur, substitué dans les effets de la promesse de vente dont ils étaient les bénéficiaires un autre acquéreur, moyennant l'engagement de celui-ci de leur verser une commission lors de la réalisation de la vente ${ }^{31}$. De même, a intérêt à demander l'annulation de la décision de préemption, alors même que le propriétaire avait renoncé à la

\footnotetext{
28 J-F. STRUILLOU, « La contestation d'une acquisition foncière publique », Études foncières, Janv-Fév. 2001, n89, p. 32.

${ }^{29}$ CAA Lyon, 8 juin 2010, req. N 09LY00319, Cne de Bluffy : BJDU 5/2010, p. 398 ; JurisData n ${ }^{\circ} 2010-012062$.

${ }^{30}$ CE, 16 déc. 1994, Cne de Sparbach : Rec. CE 1994, p. 544 ; BDJU 6/1994, p. 79, Obs. J-C Bonichot ; Dr. Adm. 1995, $\mathrm{n}^{\circ} 446-3$, Obs. C.M.

${ }^{31}$ CE, 30 Juillet 1997, Sté nouvelle Etude Berry et Attali, cité in in René. HOSTIOU et Jean-François. STRUILLOU, Expropriation et préemption, op cit,p. 623.
} 
transaction, le requérant qui détient un droit de préférence sur l'immeuble, car cette annulation aura pour effet de donner son plein et entier effet au pacte de préférence qui lui avait été consenti par le propriétaire ${ }^{32}$.

Comme autre exemple attestant de l'action du juge administratif en matière de protection de la propriété foncière préemptée, l'on citerait notamment sa contribution à la résolution du problème lié au déclenchement du délai de recours contentieux contre une décision de préemption illégale. Pour solutionner cette difficulté, le juge administratif a fait recours à la théorie de la connaissance acquise largement répandue en droit commun du contentieux administratif. En effet, elle stipule qu'en l'absence de publicité régulière, c'est la connaissance de fait de la décision administrative par les intéressés qui va déclencher pour ces derniers le délai du recours contentieux ${ }^{33}$. Appliquée au contentieux de la préemption foncière, il a ainsi été jugé que si le délai de recours contentieux à l'encontre d'une décision de préemption ne court en principe à l'égard de l'acquéreur évincé, qu'à compter de sa notification à ce dernier avec indication des voies et délai de recours, cette règle est néanmoins écartée lorsque l'acquéreur évincé a manifesté sa connaissance de l'existence et du contenu même de la décision litigieuse, ainsi que des voies et délais de recours contentieux, cette connaissance étant, selon la jurisprudence, suffisante pour faire courir le délai de recours à son égard ${ }^{34}$.

Ces exemples et bien d'autres témoignent de ce que le juge administratif joue désormais un rôle majeur dans la cadre du contentieux de la péremption foncière. Le juge judiciaire n'a plus l'entier monopole de la compétence en la matière. Toutefois cette consécration du juge administratif comme protecteur à part entière de la propriété foncière laisserait entrevoir une concurrence avec son homologue le juge judiciaire. En réalité, il s'agit non pas d'une concurrence qui n'aurait pas de sens et qui serait préjudiciable aux administrés comme à l'administration, mais d'une véritable «Cohabitation» qui peut déboucher sur un dialogue des juges. Au demeurant, le sacre du juge administratif comme protecteur de la propriété foncière est désormais acté, ainsi donc, il sera mieux à même à assurer sa mission de contrôle de la légalité des décisions de préemption foncière.

\section{B) Le contrôle de la légalité des décisions de préemption foncière par le juge administratif}

Désormais considéré comme un fervent défenseur de la propriété foncière face au droit de préemption, le juge administratif ne manque pas à l'occasion d'un contentieux d'effectuer des contrôles de légalité des décisions de préemption afin d'éviter des abus. Le législateur a clairement circonscrit le domaine de contrôle de la légalité des décisions de préemption foncière (1). Ce travail réalisé, on pourrait aisément en tirer les conséquences qu'induisent le dit contrôle (2).

\footnotetext{
${ }^{32}$ CE, 20 mars 1991, Roucaute : Dr.adm. 1991, nº 260.

${ }^{33}$ René. CHAPUS, Droit du contentieux administratif, Montchrestien, 13é éd. 2008, n 721 et S, p. 645.

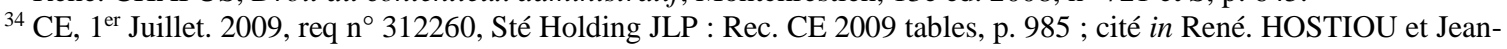
François. STRUILLOU, Expropriation et préemption, op cit, p. 634.
} 


\section{1) La délimitation du domaine de contrôle de la légalité des décisions de préemption foncière}

La délimitation du champ de contrôle de la légalité des décisions de préemption par le juge administratif n'a pas toujours été aisée car, le contentieux de la préemption foncière a toujours posé d'énormes problèmes liés à la répartition des compétences entre les ordres de juridictions administratives et judiciaires, puisque les limites ne sont pas assez étanches. Néanmoins, le juge administratif, par témérité à tant bien que mal essayé de circonscrire un domaine de contrôle de la légalité des décisions de préemption foncière. Ainsi, ce champ intègre d'abord la vérification du caractère réel des projets justifiant la mise en œuvre du droit de préemption. En effet, il est exigé de la part de collectivités territoriales décentralisées (C.T.D) qui souhaiteraient préempter des espaces fonciers de pouvoir justifier que les projets qui les amènent à recourir à cet instrument juridique sont effectivement réels et précis. Cette exigence a pour ainsi dire constituer une garantie contre la toute-puissance que confère la préemption foncière à l'Etat mais aussi aux C.T.D. Elle contrecarrerait par-là, le recours à des projets fantaisistes, imaginaires ou encore inexistants. Cette obligation du caractère réel des projets pouvant justifier le recours au droit de préemption, n'est pas que porteuse de vertu dans le cadre de la protection du droit de propriété face au droit de préemption, elle pourrait être aussi considéré comme une entrave à l'action des C.T.D ou de l'Etat puisque d'après l'esprit de ce principe, il faut justifier d'un projet certain et précis. En droit comparé, le Conseil de l'Etat français qui a particulièrement critiqué ce principe ${ }^{35}$, estimait qu'il était difficile, voire incongru, d'exiger d'une collectivité qu'elle justifie d'un projet à la date d'un événement qui, bien souvent, était l'élément déclencheur de l'opération ${ }^{36}$.

Aussi, le juge administratif ${ }^{37}$ s'assure que la décision de préemption a suffisamment été motivée $^{38}$. En effet, la motivation est une exigence de pure forme qui oblige l'auteur de la décision à en exprimer les motifs dans un document écrit. Appréciant, le caractère fondamental de la motivation de la décision de préemption en matière foncière, le juge administratif ne se prive pas de son droit d'exiger une motivation complète et précise de la décision qui doit indiquer l'opération pour laquelle le droit est mis en œuvre. Il soutient qu'est illégale la décision qui met en œuvre le droit de préemption urbain en vue de constituer des réserves foncières pour favoriser des activités économiques, sans aucune précision sur l'opération. L'obligation de motivation des décisions de préemption est une compétence liée et en même temps une condition de légalité des décisions concernées, dont l'observation est

\footnotetext{
${ }^{35}$ Ces critiques faites par le Conseil d'Etat allaient trouver leur issue jurisprudentielle avec l'arrêt Commune de Meung-surLoire, aux termes duquel «les collectivités titulaires du droit de préemption urbain peuvent légalement exercer ce droit, d'une part, si elles justifient, à la date à laquelle elles l'exercent, de la réalité d'un projet d'action ou d'opération d'aménagement répondant aux objets mentionnés à l'article L. 300-1 du code de l'urbanisme, alors même que les caractéristiques précises de ce projet n'auraient pas été définies à cette date, et, d'autre part, si elles font apparaître la nature de ce projet dans la décision de préemption $»^{35}$. Le Conseil d'État semble desserrer, par cette jurisprudence, les mailles de son contrôle quant à la justification de la réalité du projet en vue duquel le droit de préemption est exercé

${ }^{36}$ Cette haute institution affirmait à ce sujet que «C'est la mise en vente du bien qui cristallise l'intention de la collectivité de l'acquérir à des fins d'intérêt général, et non l'existence d'un projet prédéfini. Bien souvent le projet, dans les limbes de l'action de la collectivité, se matérialise grâce à la déclaration d'intention d'aliéner ». Voir J-F. STRUILLOU, « Motifs et motivation de la décision de préemption », A.J.D.A, 2008, p. 1449.
}

${ }^{38}$ V. CAA. Marseille, 31 mai 2001, commune d'Eygalieres, req. n 98 MA01527. 
placée sous le contrôle du juge ${ }^{39}$. Au final, le contrôle de la légalité des décisions de préemption opéré par le juge administratif produit de conséquences juridiques.

\section{2) Les conséquences du contrôle de la légalité des décisions de préemption foncière}

Le contrôle de la légalité des décisions de préemption pourrait déboucher sur l'annulation des actes de préemption pris par la personne publique préemptrice (a). Cette annulation entrainerait la mise en jeu de la responsabilité du titulaire du droit de préemption (b).

\section{a) L'annulation de la décision de préemption foncière}

L'annulation de la décision de préemption pourrait être prononcée par le juge administratif avant le transfert de propriété. Ainsi, il est prévu dans cette hypothèse que, le propriétaire retrouve temporairement, pour une seule année, la faculté de vendre son bien librement sans limite posée par le droit de préemption foncière. Même si ce délai reste relativement court, le législateur a souhaité mettre un terme à la paralysie qui affectait le propriétaire-vendeur et qui pouvait être victime de la mauvaise foi du titulaire du droit de préemption urbain ${ }^{40}$. Bien plus, «Si l'annulation de la préemption est prononcée avant tout transfert de propriété, le titulaire du droit de préemption ne peut exercer son droit à nouveau sur le bien en cause pendant un délai d'un an à compter de la décision juridictionnelle devenue définitive. Passé ce délai d'un an, le propriétaire peut vendre son bien à l'acquéreur évincé ou à un tiers sans souscrire une nouvelle déclaration d'intention d'aliéner, à la condition toutefois que la vente se fasse aux prix et conditions de la déclaration initiale. Dans certains cas, le propriétaire est tenu de vendre son bien à l'acquéreur évincé, lorsque ce dernier détient, du fait de l'avant-contrat, des droits sur ledit bien ${ }^{41}$. Réciproquement, l'acquéreur initial peut être, selon le contenu de la promesse de vente, tenu d'acquérir le bien ${ }^{42}$, dans ce cas, le propriétaire lésé n'est pas tenu par les prix et conditions qu'il avait mentionnés dans la déclaration d'aliéner ${ }^{43}$.

Au cours du même contrôle de la légalité de la décision de préemption par le juge administratif, il est possible que l'annulation de celle-ci intervienne après le transfert de propriété au profit de la personne publique préemptrice. Dans cette hypothèse, la situation est plus délicate, car l'annulation de la décision d'exercer le droit de préemption est, sans effet sur la validité du contrat conclu entre le vendeur et le titulaire du droit de préemption ${ }^{44}$ et n'a pas, par elle-même, pour effet de mettre fin au droit de propriété de son titulaire sur le bien concerné $^{45}$. La jurisprudence s'était attachée à mettre en place un protocole visant à rétablir le vendeur et l'acquéreur évincé dans leurs droits en tirant les conséquences du caractère rétroactif de l'annulation de la décision de préemption ${ }^{46}$. En droit comparé, la jurisprudence

\footnotetext{
${ }^{39}$ CE, 30 juillet 1997, commune de Montreuil-sous-Bois, rec., p. 309.

${ }^{40}$ L. PEREZ, Droit de préemption urbain : à la recherche d'un équilibre entre droit de propriété et puissance publique, Rapport de stage, Université Lyon Jean Moulin, Faculté de Droit, 2014 - 2015, p. 65.

${ }^{41}$ Cass. 3e civ, 13 juill. 1994, Cts Giroud c/ Cne Échirolles et a : D. 1995, somm. p. 160, obs. P. Carrias.

${ }^{42}$ Cass. 3e civ, 9 nov. 1999, Sauzière c/ Lanoue, : AJDI 2000, p. 632, obs. A. Lévy.

${ }^{43}$ V. également P. BENOIT-CATTIN, « Le contentieux de l'urbanisme après la loi Bosson du 9 février 1994 », JCP 1994, éd. N, I, p. 27 ; J-L Lala et Y.-M. Doublet, « La loi n 94-112 du 9 février 1994 portant diverses dispositions en matière d'urbanisme et de construction », Petites affiches, 29 avril 1994, n 51, p. 6. - C. Jamin, « Législation française en matière de droit privé », RTD civ. avril-juin 1994, p. 432.

${ }^{44}$ CE 21 avr. 2000, Sté Foncier Immobilier lyonnais, n 180325, rec. T. 903 ; BJDU 2000. 183, concl. F. Lamy.

${ }^{45}$ CE 17 mai 2006, Commune de Jonquières, $n^{\circ} 281509$, Rec. T. 734.

${ }^{46}$ CE, sect., 26 février 2003, Bour, n ${ }^{\circ} 231558$, rec. 59 ; BJDU 2203. 106, concl. P. Fombeur ; BJCL 2003. 710, nos obs. ; Defrénois 2003. 1336, note J.-Ph. Meng ; RDI 2003. 379, obs. P. S.-C. ; JCP-A 2003. 1900, note Ph. Billet; AJDA 2003 14 avr. 2003. 729, chron. F. Donnat et D. Casas ; BJDU 2003. 168, obs. É. FATÔME, « Pour une mise en œuvre encore récente de cette jurisprudence », cf. CAA Marseille, 10 juillet 2015, SARL Le Val de Provence, n ${ }^{\circ}$ MA04008.
} 
Bour a été pour l'essentiel reprise par le législateur dans la cadre des différentes réformes législatives récentes. Ainsi, il est désormais prévu, qu'en cas d'annulation ou de déclaration d'illégalité d'une décision de préemption postérieurement au transfert de propriété au profit de la collectivité publique locale préemptrice, celle-ci doit en proposer la rétrocession à l'ancien propriétaire et, en cas de refus, à l'acquéreur évincé si son nom figurait dans la déclaration d'aliéner. Cette proposition doit être faite à un prix visant à rétablir, sans enrichissement injustifié de l'une des parties, les conditions de la transaction à laquelle l'exercice du droit de préemption a fait obstacle. Et le code précise enfin qu'en cas de désaccord, le prix sera fixé par le juge de l'expropriation ${ }^{47}$. Si jamais le juge constate l'illégalité de la décision de préemption, la responsabilité du titulaire du droit de préemption foncière pourrait être établie.

\section{b) La mise en jeu de la responsabilité du titulaire du droit de préemption}

L'atténuation des abus perpétrés par le droit de préemption sur la propriété foncière ne peut être effective notamment que par le pouvoir du juge administratif de mettre en jeu la responsabilité du titulaire du droit de préemption, en cas de violation des règles régissant la préemption foncière ${ }^{48}$. Cette responsabilité de l'administration est assurément la résultante d'une faute qui constitue en quelque sorte son fondement ${ }^{49}$. L'illégalité dans l'exercice du droit de préemption foncière constitue, en application des principes traditionnels de la responsabilité publique, une faute susceptible d'engager la responsabilité de la personne publique à l'égard notamment du propriétaire foncier ${ }^{50}$, laquelle faute aurait directement causé un préjudice certain ${ }^{51}$.

Au-delà de la jurisprudence quasi constante au sujet de la faute comme fondement de la responsabilité, peut-on admettre l'hypothèse d'une responsabilité sans faute du titulaire du droit de préemption? À cette question, la jurisprudence administrative apporte une réponse pertinente, en décidant de rejeter la possibilité d'une responsabilité sans faute du titulaire du droit de préemption vis-à-vis d'un promoteur immobilier qui se proposait d'acquérir les immeubles préemptés. La jurisprudence a, en effet, considéré que la mise en œuvre du droit de préemption ne saurait occasionner à l'égard de l'acquéreur évincé -qui se proposait d'acquérir les parcelles en vue de la réalisation d'un ensemble immobilier- des sujétions ou aléas «excédant ceux que doivent normalement supporter les vendeurs ou les acquéreurs de terrains situés en zone urbaine ${ }^{52}$. Bien que l'élément primordial de la responsabilité soit établit, il faut encore pouvoir démontrer la réalisation du préjudice. Cette exigence est satisfaite lorsqu'il ressort de l'ensemble des circonstances de l'affaire et, en particulier de l'acte conclu entre le propriétaire et l'acquéreur évincé, que la réalisation de la vente initiale était probable. Aussi, lorsque le propriétaire foncier est en mesure de prouver l'existence d'un engagement ferme d'acquisition du bien aux prix et conditions fixés dans la déclaration

\footnotetext{
${ }^{47}$ Sur les distances prises avec la jurisprudence Bour, cf. Pierre. SOLER-COUTEAUX et Elise. CARPENTIER, Droit de l'urbanisme, HyperCours, Dalloz, 2015, § 996 et s.

${ }^{48}$ Voir. Désiré-Aurèle. MBANG ESSONO, Le droit de préemption en matière foncière, thèse de Doctorat précitée, p. 146.

${ }^{49}$ René. HOSTIOU et Jean-François. STRUILLOU, Expropriation et préemption, op cit, Expropriation et préemption, op cit, p. 679.

${ }^{50}$ CE, 10 fev. 2016, $n^{\circ}$ 382293, Cne d'Aubervilliers c/ Alteagroup Real Estate Sarl : JurisData n ${ }^{\circ}$ 2016-002146. -CAA Versailles, 30 déc. 2013, $n^{\circ} 12$ VE00664, Sté MK Immo : juris data ${ }^{\circ}$ 2013-033326. - CAA Versailles 30 . 2014, $n^{\circ}$ 13VEE00956, Sté Alteagroup Real Estate Sarl : Juris Data n² 2014-010012.

${ }^{51}$ CAA Nantes, 30 avril. 2014, ${ }^{\circ} 12$ NT02263, Feray C/ Cté de cnes du Vouvrillon.

${ }^{52}$ CE, 7 mai 1986, SA Etudes Malesherbes : Rec. CE 1986, tables, p. 78 ; D. 1987, inf. rap. P. 117 ; RD Imm. 1986, p. 456, chron. Gaudemet (y.) et Labetoulle.
} 
d'intention d'aliéner, le propriétaire empêché de vendre du fait d'une décision illégale de préemption a droit à une indemnité ${ }^{53}$. L'obligation de démontrer la réalisation du préjudice demeure insuffisante car, il va falloir encore établir le lien de causalité entre la faute et le préjudice subi par le propriétaire-vendeur du bien préempté. Il s'agit ici de tout faire pour ne pas engager la responsabilité d'un individu pour des faits dont il n'a été de loin ou de près l'auteur. Cette exigence légale semble pouvoir protéger le titulaire du droit de préemption foncière, car il ne peut être tenu pour responsable des dommages dont il n'est pas l'auteur, et c'est bien justice. Comment pouvait en être autrement d'engager la responsabilité du titulaire du droit de préemption pour une décision de préemption illégale qui n'émane pas de son action $^{54}$. Si ce lien de causalité est établi, la personne publique fautive pourrait être astreinte à la réparation du préjudice subi par le propriétaire du bien préempté. Si ce lien de causalité est établit, le propriétaire-vendeur du bien préempté pourrait bénéficier d'une indemnisation car selon J. Ghestin ${ }^{55}$ «L'exercice fautif $d u$ droit de préemption engendre une obligation à réparation du préjudice subi par le propriétaire du bien préempté ${ }^{56}$. Au demeurant, la compétence juge administratif à connaitre du contrôle des opérations de préemption foncière est désormais un acquis en jurisprudence. Le juge judiciaire camerounais est aussi compétent pour assurer la protection de la propriété foncière confrontée au droit de préemption.

\section{II) Le cantonnement du domaine de compétence du juge judiciaire en matière de protection de la propriété foncière opposée au droit de préemption}

Pendant la mise en œuvre du droit de préemption par l'Etat ou par les C.T.D, le juge judiciaire veille au respect scrupuleux des textes légaux encadrant les opérations de préemption foncière. "Tout œuvre humaine n'étant pas parfaite » comme le dit l'adage, l'on est souvent appelé à constater que les autorités chargées d'exécuter les opérations de préemption foncière, se mettent en marge de la norme, soit par inadvertance ou alors de mauvaise foi. Ainsi donc, grâce à l'action du juge judiciaire, on est certain que de tels dérapages seront désormais réduits à leur portion congrue. Cette action du juge judiciaire vise au fond, à assurer sa mission naturelle de gardien de la propriété foncière (A). Bien plus, il assure Cahin caha le contrôle de la légalité des opérations de préemption foncière (B).

\section{A) Le juge judiciaire, gardien naturel de la propriété foncière dans le cadre de la mise en œuvre du droit de préemption}

Le droit camerounais réserve en matière de protection de la propriété foncière, une place particulière au juge judiciaire. Car, il contribue par son office à contrer toute atteinte dont pourrait être victime la propriété foncière dans son essence. Aujourd'hui cette règle que l'on qualifierait de dogme juridique demeure applicable, puisqu'elle a consacré le juge judiciaire au rang de gardien de la propriété privée ${ }^{57}(1)$. Cette consécration a reçu un ancrage au plan juridictionnel (2).

\footnotetext{
${ }^{53}$ CAA Lyon, 13 juillet. 1993, Mme Thobie Vve de Guidis. CAA Paris, 30 aout 1996, n 95PA02794, Cne de Montreuil c/ Laporte, confirmé par CE, 28 juill 1999, Cne de Montreuil-sous-Bois c/ Cts Laporte : B.D.J.U 5/1999, p. 367.

${ }^{54}$ CE, 21 juin 2006, M. Gantois : JurisData n ${ }^{\circ}$ 2006-070402 ; BJDU 4/2006, p. 284 concl. J-H. Stahl.

55 Jacques. GHESTIN, Traité des contrats, L.G.D.J 1994, p. 456.

${ }^{56}$ CE, 15 mai 2006, commune de Fayet, Rec. CE, p, 250 ; BJDU 4/2006, p. 267, concl. J. H. Stahl, obs. J. cl. B ; A.J.D.A 2006, p. 2240, obs. B. JORION; p. 1071, obs. S. Brondel cités in J-F. STRUILLOU, « Responsabilité du fait d'une décision de préemption illégale prise par la commune », commentaire sur CE, 7 mai 2015, req $\mathrm{n}^{\circ} 371915$, xc/ commune de Dreux, jurisdata $\mathrm{n}^{\circ}$ 2015-010273, in R.T.D.I ${ }^{\circ}$ 3-2015, p. 12

${ }^{57}$ Civ. $3^{\circ}, 30$ mars 1994, n 91-22240, Civ. $3^{\circ}, 14$ mai 1997, n 95-16230, Civ. $3^{\circ}, 12$ juil 2000, n 98-22866, Cts de SaintPern. Citées in G. GANEZ-LOPEZ, « Droits de préemption : Droit de préemption urbain Zones d'aménagement différé,
} 


\section{1) La consécration du juge judiciaire comme gardien de la propriété foncière}

Parce que le droit de propriété foncière avait été considère comme étant un moyen par lequel l'homme ne peut s'épanouir en toute dignité au sein de la société, et aussi par une volonté de mettre ce droit fondamental à l'abri des atteintes de divers ordres, il a paru important de lui donner une assisse légale. C'est ainsi que très tôt, la loi du 8 mars 1810 et de ses réformes successives $^{58}$ relative à l'expropriation pour cause d'utilité publique va disposer la première que le juge judiciaire détient cette vocation naturelle de gardien de la propriété privée ${ }^{59}$. Le juge judiciaire va donc t'être reconnu compétent pour connaitre notamment des questions liées au transfert de propriété à la suite d'une expropriation ${ }^{60}$. Par cette consécration, le juge judiciaire serait amené à sanctionner en toute indépendance les dérives de pouvoir perpétrées par la puissance publique préemptrice. C'est assurément cette fermeté qu'avait fait montre le juge judiciaire face aux atteintes réalisées sur le droit de propriété que la majeure partie de la doctrine sera poussée à penser qu'étant pas très proche de l'administration, il serait plus à même à protéger les propriétaires privés victimes des abus de l'administration au cours de la procédure de préemption. C'est donc dire qu'au regard du droit de propriété, la compétence du juge judiciaire apparait comme la résurgence d'une époque où le juge administratif faisait l'objet d'un déficit manifeste de confiance de la part des citoyens. Cette défiance, déjà dénoncée dans la note dite de SchÖnbrunn adressée par Napoléon Bonaparte à Cambaceres, se retrouve dans la loi du 8 mars $1810^{61}$. À la suite de cette loi du 8 mars 1810 , le Commissaire du Gouvernement Romieu indiquait dans ses conclusions au Recueil Lebon sous l'arrêt Consorts Montlaur, Mary c/ Balmigère, maire de Tournessan en 1904, qu'" aucun doute n'est possible : les questions de propriété et de possession relèvent de l'autorité judiciaire $»^{62}$.

Dans le même sens, plusieurs autres lois seront adoptées par le législateur camerounais à l'effet de donner davantage d'importants pouvoirs au juge judiciaire devant lui permettre d'assurer l'encadrement juridique de la préemption foncière. En réalité, la volonté du législateur est de préserver les droits de propriétaires des biens soumis à préemption qui sont le plus souvent victimes des affres de l'action foncière des collectivités territoriales décentralisées notamment. Puisque, dans l'optique d'assurer une mise en œuvre efficiente des politiques publiques au niveau local, les pouvoirs publics locaux ont généralement recours au droit de préemption à l'effet de leur permettre d'avoir accès aux espaces fonciers situés tant en milieu urbain que rural. Il est désormais clair que le juge judiciaire est le gardien naturel de la propriété foncière. Ce principe de droit a reçu un ancrage jurisprudentiel.

Espaces naturels sensibles des départements, Droit de priorité, Droits de délaissement », in EXPRO Express, Décembre 2010, p.17.

${ }^{58}$ Jean-François. STRUILLOU, Protection de la propriété privée immobilière et prérogatives de puissance publique, th. Nantes, Préf. R. HOSTIOU, L’Harmattan, 1996, p. 266.

${ }^{59}$ L. PEREZ, Droit de préemption urbain : à la recherche d'un équilibre entre droit de propriété et puissance publique, Rapport de stage précité, p.52.

${ }^{60}$ C. LAVIALLE, ajoute les lois révolutionnaires des 4 septembre 1792 et 14 ventôse an II, in Le juge administratif et l'exception de propriété, RFDA 2004, p. 497.

${ }^{61}$ Jacqueline. MORAND DEVILLER, Cours de droit administratif des biens, p. 383. Dans cette note, en date du 29 septembre 1809, Napoléon considère notamment que l'une des garanties à offrir aux citoyens expropriés est de reconnaitre la compétence du juge judiciaire en matière de transfert et de fixation de l'indemnité. J'en arrive à ce principe, c'est qu'aucun citoyen ne peut être exproprié que par acte judiciaire ou acquiert la propriété par testament par donation ou par achat. Tous ces actes sont des actes judiciaires ; on ne doit prendre la propriété que part une vente ou une sentence qui soient également des actes judiciaires » (Extraits).

${ }^{62}$ T. confl., 24 déc. $1904, \mathrm{n}^{\circ} 581:$ Rec. CE, p. 888-891, p. 889. 


\section{2) Les bases jurisprudentielles d'un principe encré en droit camerounais}

Le juge est un gardien du droit et des libertés publiques au sein d'une société. Cette mission exaltante du juge n'a pas épargné l'un des droits fondamentaux que l'homme dispose à savoir le droit de propriété foncière. En effet, la jurisprudence a très tôt reconnu au juge judiciaire un pouvoir quasi absolu de veiller à la protection du droit de propriété face à la toutepuissance de l'administration. C'est ainsi que le 6 novembre 1872, la Cour de cassation déclare que "l'autorité judiciaire (est la) gardienne traditionnelle de la propriété en cas d'atteintes administratives irrégulières ${ }^{63}$. Emboitant le pas de la haute juridiction judiciaire, le Tribunal de conflits va de façon sentencieuse soutenir que «La protection de la propriété privée rentre essentiellement dans les attributions de l'autorité judiciaire ${ }^{64}$. L'on constate dans ces cas de jurisprudence que l'unanimité demeure perceptible quant à la reconnaissance $\mathrm{du}$ juge judiciaire comme garant du bon épanouissement de la propriété foncière des particuliers.

Parlant plus spécifiquement de la compétence du juge judiciaire dans le cadre de l'encadrement juridictionnel du droit de préemption foncière, la jurisprudence a de façon remarquable reconnu un espace de compétence au juge judiciaire, question de lui permettre de s'assurer de la bonne protection de la propriété foncière face au droit de préemption. C'est ainsi que la Cour de cassation a aussi jugé, par exemple, que la vérification du dépôt de la décision de préemption avant l'expiration du droit de préemption n'impliquait aucune difficulté sérieuse quant à la validité de l'acte administratif en cause et rentrait dans la compétence du juge judiciaire ${ }^{65}$. Bien plus, dans l'arrêt Soyer ${ }^{66}$ le juge conclut à la compétence du tribunal de grande instance «de par la vocation naturelle et traditionnelle de ce dernier en matière d'atteinte ou de contestation relative au droit de propriété ou à ses démembrements ». Dans toutes les premières décisions des juges du fond attribuant compétence aux juridictions judiciaires se trouve de façon sous-jacente cette motivation ${ }^{67}$. En effet, la préemption fait intervenir un certain nombre d'acteurs qui sont, directement ou indirectement, touchés par la préemption.

Quant à M. Morange, il constatait dans son commentaire des arrêts du Conseil d'Etat en matière du droit de préemption le principe même d'une compétence judiciaire. Tout en remarquant la substitution de la compétence à la compétence administrative dans le cas de la voie de fait et d'emprise sur la propriété immobilière, il invoque l'absence de toute voie de fait et s'agissant de l'emprise, il ajoute qu'il est de la compétence des tribunaux administratifs de constater son irrégularité. Par conséquent ses juridictions seraient seules aptes à affirmer l'irrégularité de l'emprise résultant du droit de préemption ${ }^{68}$. Ce sont les juges judiciaires qui sont gardiens du respect du droit de propriété et, même s'ils interprètent souvent restrictivement le droit de préemption au détriment de l'intérêt général, on ne saurait leur retirer leur vocation à connaitre des faits d'emprise sur l'immeuble. C'est désormais clair, le

\footnotetext{
${ }^{63}$ G. SIMON, Le juge judiciaire, gardien de la propriété privée immobilière, Bibliothèque des thèses, Mare\&Marin, 2011, p. 497.

64 T.C. 4 juin, 1940, Recueil Lebon, p. 248.

${ }^{65}$ Civ. $3^{\circ}, 18$ oct. $1994, \mathrm{n}^{\circ} 92-18142$.

${ }^{66}$ Paris, 13 mars 1967. D. 1965. 52, note Chesne (G.), citée in Corine. SAINT-ALARY-HOUIN, Le droit de préemption, op cit, p. 365.

${ }^{67}$ Voir TGI, Cahors, 12 mai 1967. D. 1968. 256, note Chesne (G.), citée in Corine. SAINT-ALARY-HOUIN, Le droit de préemption, op cit, p. 365.

${ }^{68}$ CE. 22. Déc. 1950. D. 1951. 548, note Morange (G.), citée in Corine. SAINT-ALARY-HOUIN, Le droit de préemption, op cit, p. 366.
} 
juge judiciaire est reconnu comme un maillon important de la protection de la propriété foncière face au droit de préemption. La juge judiciaire camerounais devrait pouvoir s'appuyer sur cette jurisprudence à l'effet de jouer sa partition, car il n'en a pas encore véritablement pu imprimer ses marques, étant donné que la notion est encore quasi méconnue de la pratique au Cameroun. L'on a fort espoir qu'il s'inspirera mutatis mutandis de l'action judiciaire de son homologue français qui a déjà une grande longueur d'avance, mais aussi une forte expérience en la matière. Le juge judiciaire s'emploi à assurer un contrôle de la légalité des décisions de préemption.

\section{B) La compétence du juge judiciaire en matière de contrôle de la légalité des décisions de préemption foncière}

Pour assurer la protection diligente de la propriété foncière préemptée, la jurisprudence reconnait de façon unanime au juge judiciaire le pouvoir d'effectuer le contrôle de la légalité des décisions de préemption (1). Toutefois, cette compétence demeure limitée (2).

\section{1) L'admission de la compétence du juge judiciaire en matière de contrôle de la légalité des décisions de préemption foncière}

De façon presque unanime, la jurisprudence a admis la compétence du juge judiciaire à connaitre des litiges liés à la légalité des actes administratifs pris dans le cadre de la mise en œuvre du droit de préemption foncière. Cette reconnaissance s'est faite de façon exceptionnelle car, le juge administratif continue à bénéficier de ce champ de compétence. En droit comparé, le Conseil d'Etat affirmera à ce sujet que «c'est aux tribunaux de l'ordre judiciaire, en l'absence d'une disposition législative donnant compétence en cette matière à la juridiction administrative qu'il appartient de connaitre des litiges relatifs au droit de préemption et cela en raison de l'atteinte grave qu'il porte au droit de propriété (...) ${ }^{69}$. Deux raisons peuvent être avancées étroitement imbriquées pour expliquer la solution retenue.

La première est liée au souci de la jurisprudence de concilier les principes régissant la répartition des compétences en matière d'appréciation de la légalité des décisions administratives prises dans le cadre de l'exercice du droit de préemption avec "l'exigence d'une bonne administration de la justice ${ }^{70}$. Il s'agit là de tempérer les inconvénients liés au dualisme juridictionnel et d'éviter qu'une application par trop rigide des principes de répartition des compétences n'oblige le juge judiciaire à surseoir à statuer jusqu'à ce que le juge administratif se soit prononcé sur la légalité de l'acte contesté, alors que la solution de l'exception d'illégalité s'impose avec force d'évidence au juge du principal. L'idée est donc ici, de restreindre encore un peu plus l'obligation de recourir aux questions préjudicielles, ce mécanisme quelque peu sophistiqué constituant toujours pour le justiciable une source de complications, d'allongement des procédures et de renchérissement de leur coût. La complexité et la lourdeur de ce système présentent en outre l'inconvénient de faciliter, voire même de favoriser, les comportements dilatoires, le titulaire du droit de préemption pouvant être tenté de jouer avec les difficultés pour le requérant d'identifier le «bon interlocuteur», susceptible de lui apporter une réponse adéquate en temps utile ${ }^{71}$.

\footnotetext{
${ }^{69}$ CE. 22 déc. 1950. D. 1951. 547, note Morange.

${ }^{70}$ René HOSTIOU et Jean-François STRUILLOU, Expropriation et préemption, op cit, Expropriation et préemption, op cit, p. 619.

${ }^{71}$ René HOSTIOU et Jean-François STRUILLOU, Expropriation et préemption, op cit, p. 618.
} 
Quant à la seconde justification, elle tient à la volonté de la Haute juridiction d'éviter que l'exigence touchant au respect des délais raisonnables, composante du droit à un procès équitable garanti par l'article $6 \S 1$ de la Convention européenne des Droits de l'Homme, ne vienne perturber le contentieux du droit de préemption. Ce danger a été très bien mis en lumière par le Commissaire du gouvernement dans ses conclusions sur l'arrêt rapporté. Après avoir observé que huit années après la décision initiale de préemption, cinq voies de recours avaient été exercées par le titulaire de ce droit, dont quatre, à ce jour sans succès, M. Girard souligne à juste titre que «le débat ouvert sur la renonciation à préemption de la propriété prête le flanc à une critique évidente sur le caractère excessif de la durée de la procédure qui pourrait se retourner, à terme, contre la Communauté d'agglomération et les deux ordres juridictionnels actionnés ${ }^{72}$. Sont ainsi dénoncées, sans que ne soit remis en cause le dualisme juridictionnel dans son principe même, les conséquences de celui-ci en tant que facteur d'allongement du règlement du litige, l'accumulation des lenteurs consécutives à l'obligation faite au requérant de saisir successivement le juge administratif et le juge judiciaire étant susceptible d'être analysée comme une violation du droit du requérant à voir sa cause entendue dans un délai raisonnable. Ce risque parait d'autant moins théorique que dans un domaine proche, celui de l'expropriation pour cause d'utilité publique, la Cour européenne des Droits de l'Homme n'a pas hésité, à partir du constat de la durée excessive d'une procédure contentieuse intentée devant les deux ordres de juridiction, à sanctionner les dysfonctionnements consécutifs à l'intervention des juges administratif et judiciaire en ce domaine $^{73}$.

En droit comparé, le Tribunal des conflits admet la compétence du juge civil pour constater à l'occasion d'un litige relevant à titre principal de l'ordre judiciaire- l'illégalité de la décision par laquelle une personne publique renonce à exercer son droit de préemption et pour en écarter l'application, dès lors qu'il résulte d'une « jurisprudence établie » que cet acte est entaché d'illégalité ${ }^{74}$. Il n'y a donc plus lieu désormais de faire le détour par le juge administratif lorsqu'il ressort clairement de la jurisprudence que l'acte administratif contesté est manifestement illégal : le juge civil de l'action est dans ce cas habilité à apprécier luimême cette illégalité. Il y'a là une application remarquable d'une jurisprudence récente et importante du Tribunal des conflits, laquelle autorise les tribunaux judiciaires à déclarer, à l'occasion d'un litige relevant à titre principal du juge judiciaire, l'illégalité des actes administratifs manifestement irréguliers ${ }^{75}$. Le juge judiciaire, saisi du contentieux au fond, peut de la sorte constater l'illégalité de l'acte administratif querellé, à la condition toutefois que cette illégalité soit certaine et évidente au vu «d'une jurisprudence établie » du Conseil d'Etat. Cette novation procède par là même à une profonde atténuation de la jurisprudence issue de l'arrêt Steptfonds, laquelle interdisait jusqu'ici au juge judiciaire d'apprécier la

\footnotetext{
72 René HOSTIOU et Jean-François STRUILLOU, Expropriation et préemption, op cit, p. 620.

${ }^{73}$ CEDH, 21 fev. 1997, Guillemin c/ France : AJDA 1997, p. 399, note Hostiou (R.), cité in René. HOSTIOU et JeanFrançois. STRUILLOU, Expropriation et préemption, op cit, p. 618.

${ }^{74}$ T. Conflits, 16 juin 2014, ${ }^{\circ}$ C3953, Mme Semavoine c/Cté d'agglomération de la Rochelle : Rec. CE 2014, tables, p. 584 ; AJDA 2014, p. 1658, note Struillou (J-F.) ; RD imm. 2014, p. 660, obs. M. Revert ; RJDA 4/2015, n 257 ; JurisData $\mathrm{n}^{\circ}$ 2014-013620 ; JCP A 30 Juin 2014, n 26, act. 529, F. Tesson; DAUH 2015, n 19 , p. 394, chron. Struillou (J-F.) Par "jurisprudence établie", il faut très certainement entendre celle qui permet au juge civil de constater sans aucune difficulté l'illégalité de l'acte contesté, c'est à dire sans qu'il ait a soupeser, apprécier les divers éléments dont il dispose à l'issue de l'instruction, sans qu'il ait un effort intellectuel à effectuer pour dissiper les doutes et rendre sa sentence. Cités in in René. HOSTIOU et Jean-François. STRUILLOU, Expropriation et préemption, op cit,p. 617.

75 T. conflits., 17 oct 2011, SCEA du chéneau : RFDA 2011, p. 1122, concl. J-D. Sarcelet, p. 1129, note B Seiller, p. 1136, note Roblot-Troiziet A ; AJDA 2012, p. 27, chron. M. Guyomar et X. Domino ; Dr adm. Janv. 2012, n 10, note Melleray (F.), cité in in René. HOSTIOU et Jean-François. STRUILLOU, Expropriation et préemption, op cit, p. 618.
} 
légalité d'un acte administratif, et ce alors même que cette appréciation est nécessaire pour la solution du litige dont il a été saisi ${ }^{76}$. Le juge judiciaire est ponctuellement compétent dans l'appréciation de la légalité de la décision de préemption, ce qui ne fait que rendre ambigüe la répartition des compétences entre juge administratif et judiciaire. Il en va ainsi de l'appréciation du respect du délai de deux mois imposé au titulaire du droit de préemption pour répondre à la déclaration d'intention d'aliéner ${ }^{77}$. La Cour justifie cette compétence car, elle ne présente « aucune difficulté sérieuse quant à la validité de l'acte administratif individuel en cause ». Il semblerait donc qu'au regard de la jurisprudence, le juge judiciaire ne soit pas compétent pour apprécier la légalité de la décision de préemption sauf lorsque celle-ci ne pose aucune difficulté sérieuse auquel cas, le juge serait dans l'obligation de surseoir à statuer, de poser une question préjudicielle au juge administratif et de rendre sa décision en fonction de la réponse à la question préjudicielle ${ }^{78}$. Au sujet du problème des décisions de préemption notifiées en dehors du délai légal, la Cour de cassation considère que si une décision de préemption est notifiée tardivement et que cette illégalité est invoquée devant le juge judiciaire, ce dernier devra vérifier lui-même si ladite décision est effectivement intervenue hors délai sans surseoir à statuer en attendant que le juge administratif saisi se prononce sur la véracité de ce moyen ${ }^{79}$.

Le juge judiciaire est dorénavant habilité à exercer un «contrôle positif » de la légalité des actes administratifs, alors qu'auparavant il n'était autorisé qu'à filtrer la pertinence des exceptions soulevées devant lui et, par la même, n'opérait qu'un «contrôle négatif »se limitant à constater la faiblesse des moyens d'illégalité soulevés à l'encontre de ces actes ${ }^{80}$. Avec cette reconnaissance, c'est le droit de propriété qui en sort renforcé, car le juge pourra dans un délai raisonnable rendre la justice à l'effet de mettre un terme aux abus éventuellement subis du fait de l'action foncière de certaines personnes publiques camerounaises. Ainsi donc, le juge judiciaire contribuera à veiller au meilleur encadrement juridique du droit de préemption foncière, à l'effet d'asseoir un équilibre entre la satisfaction de l'intérêt général et la sauvegarde de la propriété foncière au cours de l'exercice ou de la mise en œuvre du droit de préemption. En dépit de cet adoubement du juge judiciaire comme acteur majeur du contrôle de la légalité des décisions de préemption, il n'en demeure pas moins vrai que ce pouvoir reste limité.

\section{2) Les limites du pouvoir du juge judiciaire en matière de contrôle de la légalité des opérations de préemption foncière}

De façon unanime, les tribunaux se refusent à apprécier l'opportunité de la décision de préemption foncière. Il n'est pas opportun pour les tribunaux de questionner le moment de la préemption de l'immeuble vendu ${ }^{81}$. En droit comparé du contentieux de la préemption des S.A.F.E.R. «l'opportunité de l'exercice du droit de préemption des S.A.F.E.R. échappe au contrôle judiciaire et relève des pouvoirs des commissaires du gouvernement appelés à

\footnotetext{
${ }^{76}$ T. Conflits, 16 juin 1923, Steptfonds : Rec. CE 1923, p. 498 ; s. 1923, 3, 49, note M. HAURIOU ; D. 1924, 3, 41, concl. M. Matter, citées in in René. HOSTIOU et Jean-François. STRUILLOU, Expropriation et préemption, op cit, p. 619.

${ }^{77}$ Civ. 1ère, 18 octobre 1994, Compagnie Abeilles Assurance c/ Ville de Fontenay-sous-Bois et autres, req. n ${ }^{\circ} 92-18142$.

${ }^{78}$ L. PEREZ, Droit de préemption urbain : à la recherche d'un équilibre entre droit de propriété et puissance publique, Rapport de stage précité, p.53.

${ }^{79}$ Cass. civ. 3e, 4 juillet 1984, Epoux Girault c/ Benarous et autres, Gaz. Pal., 9 mars 1985, p. 23.

${ }^{80}$ B. SEILLER, note ss T. confl. 17 oct 2011, SCEA du Chéneau : RFDA 2011, spéc. P. 1133.

81 TGI. Strasbourg, 19 janvier 1972. D. 1972, Som. 178.
} 
surveiller leurs opérations ${ }^{82}$. On sait en effet, que les S.A.F.E.R. doivent être autorisées à préempter et seuls ses organes d'autorisation apprécient la nécessité d'une préemption ${ }^{83}$.

Cette généralité de l'exclusion d'un contrôle judiciaire de l'opportunité de la décision de préemption s'explique à la fois par le refus d'apprécier l'acte administratif de préemption et par la nature du droit de préemption. En effet, en dissociant opportunité et légalité, les juridictions judiciaires évitent de se faire les juges de l'activité de l'administration. Elles n'apprécient pas si les autorités administratives ont eu raison ou tort d'engager les fonds de l'Etat dans une opération d'achat du bien préempté ${ }^{84}$. Il faut ajouter que ce refus du contrôle de l'opportunité s'explique, par la nature du droit d'option du droit de préemption, le titulaire du droit d'option peut choisir librement entre tel ou tel des parties qui lui sont proposés ${ }^{85}$.

La possibilité pour le juge judiciaire d'évincer une décision de préemption intervenue hors délai n'est pas absolue. En effet, elle trouve sa limite dans l'interprétation que fait le juge de l'acte administratif qui doit dès lors ne présenter aucune «difficulté sérieuse ${ }^{86}$ de «nature à faire naître un doute dans un esprit éclairé » ${ }^{87}$, "la question de savoir si les tribunaux judiciaires sont incompétents en raison du principe de séparation des autorités pour répondre eux-mêmes à une question d'appréciation de légalité d'un acte administratif ne se pose qu'à condition que l'appréciation de légalité se heurte à aucune difficulté sérieuse de nature "à faire naître un doute dans un esprit éclairé " ${ }^{88}$. Il en résulte qu'il n'y a pas lieu à question préjudicielle si les dispositions de l'acte administratif étaient claires et précises car, dans ce cas, il s'agit simplement pour le tribunal judiciaire "d'appliquer, et non pas d'interpréter, l'acte administratif ${ }^{89}$.

\section{CONCLUSION}

En définitive, les juges administratif ou judiciaire contribuent par leur office à la protection de la propriété foncière préemptée. Cette action devrait à coup sûr donner plus de vigueur au droit de propriété foncière, car ce droit fondamental est menacé de toute part. Pour que cet objectif de garantie juridictionnelle de la propriété foncière soit réellement atteint, l'on devrait s'assurer d'une répartition claire du champ de compétence entre les juges administratifs et judiciaires camerounais. Le législateur camerounais aurait le choix de prendre en compte la suggestion faite par le Conseil d'Etat français «d'unifier au sein de la juridiction administrative le contentieux des décisions de préemption et des contrats connexes à ces décisions aux fins de pallier les difficultés qu'engendre en la matière un double contentieux, en particulier, lorsqu'il s'agit de tirer les conséquences de l'annulation de la décision de préemption intervenue après la conclusion de la vente issue du processus de préemption ${ }^{90}$.

\footnotetext{
${ }^{82}$ Rennes, 6 mai 1970, Gaz. Pal. 1970. 2. Sem 202, note SIGNY (R.) et CADIOU.

${ }^{83}$ Corine. SAINT-ALARY-HOUIN, Le droit de préemption, op cit, p. 355.

${ }^{84}$ Corine. SAINT-ALARY-HOUIN, Le droit de préemption, op cit, p. 356.

${ }^{85}$ I. NAJJAR, Le droit d'option, contribution à l'étude du droit potestatif et de l'acte uniforme, p. 271, n²64.

${ }^{86}$ Cass. civ. 1e, 18 octobre 1994, Cie Abeille Assurance c/ Ville de Fontenay-Sous-Bois et autres, Bull. civ. n²99, p. 217.

${ }^{87}$ Cf. René. CHAPUS, Droit du contentieux administratif, op cit, p. 321.

${ }^{88}$ LAFERRIERE, Traité 2e éd., t. 1, Paris, Berger-Levrault 1987, p. 498.

${ }^{89}$ Sur la distinction parfois délicate entre la simple application et l'interprétation, cf. Cass. Soc., 17 novembre 1960, Bull. civ, IV, p. 799.

${ }^{90}$ V. rapport du Conseil d'Etat de 2008 sur le droit de préemption.
} 